\section{Estilo de vida saudável em São Paulo, Brasil}

\author{
Healthy lifestyle in São Paulo, Brazil
}

\section{Estilo de vida saludable en São Paulo, Brasil}

\section{Resumo}

O objetivo foi analisar o estilo de vida das populações adolescente, adulta e idosa do Município de São Paulo, Brasil, de acordo com variáveis demográficas e socioeconômicas. Foi realizado estudo transversal, de base populacional, com dados do Inquérito de Saúde no Município de São Paulo (ISA-Capital 2008). O estilo de vida foi definido pela avaliação da atividade física, consumo alimentar, tabagismo, consumo abusivo e dependência de álcool, de acordo com as respectivas recomendações. A prevalência de estilo de vida saudável foi de 36,9\% entre idosos, 15, 4\% entre adultos e 9,8\% entre adolescentes, sendo maior no sexo feminino entre idosos e adultos. Dentre aqueles com estilo de vida não saudável, 51,5\% dos idosos, 32,2\% dos adultos e 57,9\% dos adolescentes não atingiram a recomendação para uma dieta adequada. A prevalência de estilo de vida saudável foi maior entre idosos, seguida pelos adultos e adolescentes. O consumo alimentar foi o principal responsável pelo estilo de vida não saudável, evidenciando a importância de intervenções para a promoção do estilo de vida saudável e, principalmente, da dieta adequada.

Estilo de Vida; Atividade Motora; Consumo de Alimentos; Hábito de Fumar; Alcoolismo
Tatiane Kosimenko Ferrari 1

Chester Luiz Galvão Cesar 1

Maria Cecília Goi Porto Alves 2

Marilisa Berti de Azevedo Barros 3

Moisés Goldbaum 4

Regina Mara Fisberg 1

doi: 10.1590/0102-311X00188015

\section{Correspondência}

T. K. Ferrari

Rua Rio Branco 80, apto. 83, São Caetano do Sul, SP 09520-620, Brasil.

tferrari@usp.br

1 Faculdade de Saúde Pública, Universidade de São Paulo, São Paulo, Brasil.

2 Instituto de Saúde, Secretaria de Estado da Saúde de São Paulo, São Paulo, Brasil.

3 Faculdade de Ciências Médicas, Universidade Estadual de Campinas, Campinas, Brasil.

${ }_{4}^{4}$ Faculdade de Medicina, Universidade de São Paulo São Paulo, Brasil. 


\section{Introdução}

As últimas décadas têm representado um período de mudanças nas condições de vida e de saúde da população brasileira, que está em acentuado processo de envelhecimento, com um padrão de trabalho e lazer modificados e com profundas transformações na qualidade e quantidade dos alimentos ingeridos ${ }^{1}$. Nesse sentido, as mudanças nos perfis epidemiológico e demográfico das populações tiveram como consequência uma maior exposição dos indivíduos aos fatores de risco relacionados às doenças crônicas não transmissíveis, especialmente às doenças cardiovasculares 2,3.

As doenças cardiovasculares constituem uma das principais causas de mortalidade e incapacidade em quase todos os países, independentemente do desenvolvimento econômico 2,4,5. Além disso, as doenças cardiovasculares constituem uma das principais causas de permanência hospitalar prolongada e são responsáveis pela principal alocação de recursos públicos em hospitalizações 6 .

As doenças cardiovasculares têm origem multifatorial e estão relacionadas com fatores de risco não modificáveis, como idade, sexo e individualidade biológica; ou modificáveis, que estão relacionados com o estilo de vida 3. Dentre esses últimos, destacam-se o tabagismo, a inatividade física, o consumo abusivo e/ou dependência de álcool e o consumo alimentar inadequado 7 , sendo que os fatores de risco modificáveis são responsáveis por $80 \%$ das doenças cardiovasculares no Brasil 2,5.

Uma vez que os fatores de risco modificáveis relacionados ao estilo de vida respondem por grande parte de todas as mortes por doenças cardiovasculares no mundo, sua mensuração e monitoramento tornam-se essenciais para o planejamento de estratégias e ações para o controle do estilo de vida não saudável, prevenção de doenças e promoção da saúde. Porém, apesar desses fatores serem potencialmente relevantes na definição do perfil epidemiológico da população e estarem associados à diminuição da mortalidade precoce por doenças cardiovasculares quando estão em situação ideal ou controlada, eles não vêm sendo monitorados de modo que possam ser avaliados de forma global. Muitos estudos avaliam esses fatores de risco isoladamente 8,9,10,11,12,13,14, mas são escassos os que analisam as interações entre os mesmos.

Sendo assim, o objetivo do presente trabalho foi descrever o estilo de vida das populações adolescente, adulta e idosa do Município de São Paulo, Brasil, de acordo com o cumprimento das recomendações para atividade física, consumo alimentar, tabagismo, consumo abusivo e dependência de álcool.

\section{Métodos}

Trata-se de um estudo transversal, de base populacional, que utilizou dados da pesquisa Inquérito de Saúde no Município de São Paulo (ISA-Capital 2008), realizado no período de setembro de 2008 a fevereiro de 2009. A amostra do ISA-Capital 2008 foi obtida por amostragem probabilística, por conglomerados, em dois estágios: setores censitários e domicílios, totalizando 3.271 pessoas.

A avaliação do consumo alimentar foi realizada em uma subamostra. Dentre as 3.271 pessoas entrevistadas no ISA-Capital, havia 2.691 maiores de 12 anos de idade. Foram considerados no presente trabalho 1.652 indivíduos que possuíam dados para avaliação da atividade física, tabagismo, consumo alimentar e consumo abusivo e dependência de álcool, sendo 560 adolescentes (12-19 anos), 585 adultos (20-59 anos) e 517 idosos (60 anos ou mais). Segundo o planejamento feito, a obtenção de 576 entrevistas em cada grupo etário permitiria obter estimativas de prevalência de 50\%, com erro de amostragem de 5 pontos porcentuais para nível de $95 \%$ de confiança e efeito do delineamento de 1,5.

O estilo de vida foi determinado pela interação de cinco domínios: atividade física, consumo alimentar, tabagismo, consumo abusivo de álcool e dependência de álcool. Os indivíduos foram classificados em duas categorias: estilo de vida não saudável ou estilo de vida saudável, sendo que o estilo de vida saudável foi caracterizado pelos indivíduos ativos, com um consumo alimentar adequado (terceiro tercil), não fumantes ou ex-fumantes, com consumo não abusivo de álcool e não dependentes de álcool (CAGE negativo). Os indivíduos classificados como estilo de vida não saudável também foram classificados em categorias intermediárias, de acordo com a quantidade e o tipo de domínios não cumpridos de forma adequada. 
Para a avaliação da atividade física, foi utilizado o International Physical Activity Questionnaire (IPAQ; http://www.ipaq.ki.se, acessado em 29/Jan/2013), versão longa. O escore da prática de atividade física em minutos por semana foi calculado para a atividade física total (soma dos minutos por semana em cada um dos quatro domínios da atividade física - atividade física no trabalho, atividade física no lazer, atividade física no transporte e atividade física no ambiente doméstico), com base na soma do número de minutos de caminhadas e atividades moderadas, mais duas vezes o número de minutos de atividades vigorosas 11,15. Baseando-se no escore da prática de atividade física total em minutos por semana, optou-se por utilizar o ponto de corte de 150 minutos semanais. Foram considerados insuficientemente ativos os indivíduos que relataram prática de atividade física total semanal por tempo inferior a 150 minutos, e ativos os indivíduos que relataram prática de atividade física total semanal por tempo igual ou superior a 150 minutos 11,16 .

O consumo alimentar foi medido usando-se o método recordatório de 24 horas (R24h), seguido pelo Índice de Qualidade da Dieta Revisado (IQD-R) 17. O IQD-R é obtido por meio da soma de dez componentes da dieta e varia de 0 a 100 pontos. Quanto maior a pontuação, melhor a qualidade da dieta 17 . Neste trabalho, os indivíduos foram categorizados em tercis, de acordo com a distribuição dos escores da qualidade da dieta, sendo que o consumo alimentar foi considerado adequado quando os indivíduos estavam classificados no terceiro tercil.

Para a avaliação do tabagismo foram realizadas perguntas sobre o hábito de fumar, o tipo de tabaco e as quantidades diárias de fumo. Foram considerados tabagistas aqueles que referiram já terem fumado alguma vez, ao menos um cigarro por dia, todos os dias, durante pelo menos um mês, e fumarem atualmente. Como ex-tabagistas foram considerados os que referiram não fumar atualmente, porém em algum período da vida fumaram ao menos um cigarro por dia, todos os dias, durante pelo menos um mês.

Para a avaliação do consumo abusivo de álcool foi utilizada uma questão sobre a frequência diária do consumo de bebidas alcoólicas. O ponto de corte para definir consumo abusivo foi de 30g/dia de etanol ou mais para o sexo masculino, e $24 \mathrm{~g} /$ dia ou mais para o feminino 18 . Já para a avaliação da dependência de álcool, foi utilizado o questionário CAGE 19, capaz de detectar uma possível dependência de álcool em estudos clínicos e populacionais. O CAGE é constituído por quatro questões referentes ao anagrama: (Cut down - redução da ingesta, Annoyed by criticism - irritação, Guilty - culpa, Eye-opener - identificação de ressaca). São considerados como casos suspeitos de dependência de álcool os indivíduos que respondem afirmativamente a duas ou mais perguntas, caracterizando CAGE positivo. O consumo abusivo e dependência foram estudados separadamente, pois, o consumo abusivo está associado a consequências adversas recorrentes e significativas, sem preencher os critérios para dependência. O consumo excessivo e constante é a condição necessária para o começo da dependência, mas, na dependência, o ato de usar uma droga deixa de ter uma função social e de eventual prazer e passa a ser disfuncional. Sendo assim, ambos estão relacionados ao estilo de vida, porém são interpretados de formas diferentes.

Para a análise dos dados foram calculadas as estimativas de prevalência de indivíduos com estilo de vida saudável, segundo o sexo, a faixa etária e as variáveis demográficas e socioeconômicas; e as estimativas de prevalência de indivíduos com estilo de vida não saudável, de acordo com a quantidade e tipo de domínios não cumpridos. A significância estatística foi calculada pelo teste qui-quadrado para heterogeneidade e pelo intervalo de $95 \%$ de confiança (IC95\%). O nível de significância estatística adotado foi de 5\%. Foi utilizado o pacote estatístico Stata (StataCorp LP, College Station, Estados Unidos), que permite incorporar os aspectos referentes ao delineamento complexo da amostra - estratos, conglomerados e ponderações - por meio do módulo survey.

Os participantes ou responsáveis assinaram o Termo de Consentimento Livre e Esclarecido, sendo que o presente estudo foi aprovado pelo Comitê de Ética em pesquisa em Seres Humanos da Faculdade de Saúde Pública, Universidade de São Paulo, sob o parecer no 498.688. 


\section{Resultados}

A Tabela 1 mostra a descrição das variáveis demográficas e socioeconômicas da amostra do estudo, composta por 1.652 indivíduos, sendo 515 idosos (idade média de 70,8 anos), 582 adultos (média de 38,7 anos) e 555 adolescentes (média de 15,3 anos).

\section{Tabela 1}

Descrição da amostra de acordo com a faixa etária, variáveis demográficas e socioeconômicas. Inquérito de Saúde no Município de São Paulo (ISA-Capital 2008), São Paulo, Brasil.

\begin{tabular}{|c|c|c|c|c|}
\hline & \multicolumn{4}{|c|}{ Proporção (\%) } \\
\hline & $\begin{array}{l}\text { Idosos } \\
(n=515)\end{array}$ & $\begin{array}{l}\text { Adultos } \\
(n=582)\end{array}$ & $\begin{array}{l}\text { Adolescentes } \\
\qquad(\mathrm{n}=555)\end{array}$ & $\begin{array}{c}\text { Total } \\
(n=1.652)\end{array}$ \\
\hline \multicolumn{5}{|l|}{ Sexo } \\
\hline Feminino & 59,7 & 55,3 & 49,4 & 54,4 \\
\hline Masculino & 40,3 & 44,7 & 50,6 & 45,6 \\
\hline \multicolumn{5}{|l|}{ Escolaridade (anos de estudo) } \\
\hline $0-3$ & 32,1 & 6,6 & - & 11,1 \\
\hline $4-7$ & 32,7 & 17,1 & - & 19,9 \\
\hline $8-11$ & 26,0 & 52,8 & - & 48,0 \\
\hline 12 ou mais & 9,2 & 23,5 & - & 21,0 \\
\hline \multicolumn{5}{|l|}{ Situação conjugal } \\
\hline Casado(a)/União estável & 57,9 & 71,0 & 73,3 & 69,8 \\
\hline Solteiro(a) & 7,1 & 11,7 & 4,9 & 9,4 \\
\hline Separado(a) & 9,2 & 10,8 & 14,1 & 11,4 \\
\hline Viúvo(a) & 24,8 & 5,2 & 6,3 & 8,1 \\
\hline NS/NR & 1,0 & 1,3 & 1,4 & 1,3 \\
\hline \multicolumn{5}{|l|}{ Raça/Cor } \\
\hline Branca & 70,1 & 57,9 & 56,9 & 59,2 \\
\hline Preta & 6,5 & 7,6 & 9,8 & 8,0 \\
\hline Parda & 20,7 & 32,4 & 32,1 & 30,8 \\
\hline Outras & 2,7 & 2,1 & 1,2 & 2,0 \\
\hline \multicolumn{5}{|l|}{ Religião } \\
\hline Sem religião & 4,4 & 6,5 & 14,1 & 8,1 \\
\hline Católica & 67,5 & 53,7 & 48,2 & 54,1 \\
\hline Protestante/Evangélica & 15,0 & 22,2 & 19,9 & 20,6 \\
\hline Espírita & 3,8 & 8,4 & 3,5 & 6,6 \\
\hline Crê em Deus & 0,9 & 3,2 & 7,6 & 4,0 \\
\hline Outras & 8,4 & 6,0 & 6,7 & 6,6 \\
\hline \multicolumn{5}{|c|}{ Renda familiar mensal (salários mínimos) } \\
\hline$<1$ & 24,0 & 16,8 & 21,3 & 18,9 \\
\hline $1-4$ & 61,2 & 60,2 & 60,6 & 60,4 \\
\hline 5 ou mais & 14,8 & 23,0 & 18,1 & 20,7 \\
\hline \multicolumn{5}{|c|}{ Escolaridade do chefe da família (anos de estudo) } \\
\hline $0-3$ & 23,6 & 9,7 & 9,9 & 11,6 \\
\hline $4-7$ & 32,9 & 19,6 & 22,3 & 22,0 \\
\hline $8-11$ & 28,2 & 48,5 & 44,5 & 44,8 \\
\hline 12 ou mais & 14,3 & 20,9 & 21,8 & 20,3 \\
\hline NS/NR & 1,0 & 1,3 & 1,5 & 1,3 \\
\hline
\end{tabular}

NS/NR: não sabe/não respondeu. 
A Tabela 2 apresenta a descrição das variáveis relacionadas ao estilo de vida, mostrando que 89,2\% dos idosos, 77,2\% dos adultos e 77,2\% dos adolescentes eram ativos; 52,2\% dos idosos, 33,4\% dos adultos e $14,6 \%$ dos adolescentes estavam no terceiro tercil, representando um consumo alimentar adequado; $56,7 \%$ dos idosos, $58 \%$ dos adultos e $92,9 \%$ dos adolescentes nunca fumaram; $33,3 \%$ dos idosos, 16,5\% dos adultos e 2,6\% dos adolescentes eram ex-tabagistas; 96,4\% dos idosos, 95,5\% dos adultos e $97,7 \%$ dos adolescentes não eram dependentes de álcool; e $85 \%$ dos idosos, $62 \%$ dos adultos e $81,2 \%$ dos adolescentes não consumiam álcool de forma abusiva.

A Tabela 3 mostra que houve uma diferença estatisticamente significante na prevalência de estilo de vida saudável e estilo de vida não saudável entre os idosos, adultos e adolescentes do Município de São Paulo em 2008 ( $<$ < 0,000). A maior prevalência de estilo de vida saudável foi encontrada entre os idosos (36,9\%), seguida pelos adultos $(15,4 \%)$ e adolescentes $(9,8 \%)$.

\section{Tabela 2}

Descrição da atividade física, consumo alimentar, tabagismo, consumo e dependência de álcool, segundo o grupo etário. Inquérito de Saúde no Município de São Paulo (ISA-Capital 2008), São Paulo, Brasil.

\begin{tabular}{|c|c|c|c|c|}
\hline & $\begin{array}{c}\text { Idosos } \\
(n=515) \\
\%(I C 95 \%)\end{array}$ & $\begin{array}{c}\text { Adultos } \\
(n=582) \\
\%(I C 95 \%)\end{array}$ & $\begin{array}{l}\text { Adolescentes } \\
\qquad(n=555) \\
\%(I C 95 \%)\end{array}$ & $\begin{array}{c}\text { Total } \\
(n=1.652) \\
\%(I C 95 \%)\end{array}$ \\
\hline \multicolumn{5}{|l|}{ Atividade física global } \\
\hline Ativo & $89,2(86,1-91,7)$ & $77,2(73,5-80,5)$ & $77,2(73,4-80,5)$ & $78,8(76,8-80,8)$ \\
\hline Insuficientemente ativo & $10,8(8,3-13,9)$ & $22,8(19,5-26,5)$ & $22,8(19,5-26,6)$ & $21,2(19,2-23,2)$ \\
\hline \multicolumn{5}{|l|}{ Consumo alimentar } \\
\hline Terceiro tercil & $52,2(47,8-56,6)$ & $33,4(29,5-37,3)$ & $14,6(11,8-17,8)$ & $31,2(28,9-33,5)$ \\
\hline Segundo tercil & $33,1(29,0-37,3)$ & $36,0(32,2-40,1)$ & $31,5(27,7-35,6)$ & $34,5(32,2-36,9)$ \\
\hline Primeiro tercil & $14,7(11,8-18,1)$ & $30,6(26,9-34,5)$ & $53,9(49,6-58,1)$ & $34,3(32,0-36,7)$ \\
\hline \multicolumn{5}{|l|}{ Tabagismo } \\
\hline Nunca fumou & $56,7(52,3-61,0)$ & $58,0(54,0-62,1)$ & $92,9(90,5-95,0)$ & $66,5(64,2-68,8)$ \\
\hline Ex-tabagista & $33,3(29,1-37,5)$ & $16,5(13,6-19,8)$ & $2,6(1,4-4,2)$ & $15,3(13,6-17,1)$ \\
\hline Tabagista & $10,0(7,6-13,0)$ & $25,5(21,9-29,2)$ & $4,5(2,9-6,6)$ & $18,2(16,3-20,1)$ \\
\hline \multicolumn{5}{|l|}{ Dependência de álcool } \\
\hline CAGE negativo & $96,4(94,3-97,8)$ & $95,5(93,5-97,1)$ & $97,7(96,0-98,7)$ & $96,2(95,1-97,1)$ \\
\hline CAGE positivo & $3,6(2,2-5,7)$ & $4,5(2,9-6,5)$ & $2,3(1,3-4,0)$ & $3,8(2,9-4,9)$ \\
\hline \multicolumn{5}{|l|}{ Consumo de álcool } \\
\hline Consumo não abusivo & $85,0(81,7-88,0)$ & $62,0(57,9-66,0)$ & $81,2(77,8-84,4)$ & $69,9(67,3-72,1)$ \\
\hline Consumo abusivo & $15,0(12,0-18,3)$ & $38,0(34,0-42,1)$ & $18,8(15,6-22,2)$ & $30,1(27,9-32,3)$ \\
\hline
\end{tabular}

IC95\%: intervalo de 95\% de confiança.

\section{Tabela 3}

Prevalência de estilo de vida saudável e estilo de vida não saudável em idosos, adultos e adolescentes. Inquérito de Saúde no Município de São Paulo (ISA-Capital 2008), São Paulo, Brasil.

\begin{tabular}{lcccc}
\hline Indicador de estilo de vida & $\begin{array}{c}\text { Idosos } \\
\text { \% (IC95\%) }\end{array}$ & $\begin{array}{c}\text { Adultos } \\
\text { \% (IC95\%) }\end{array}$ & $\begin{array}{c}\text { Adolescentes } \\
\text { \% (IC95\%) }\end{array}$ & $\begin{array}{c}\text { Total } \\
\text { \% (IC95\%) }\end{array}$ \\
\hline Estilo de vida saudável & $36,9 *(32,7-41,2)$ & $15,4 *(12,6-18,7)$ & $9,8 *(7,4-12,5)$ & $16,8(15,1-18,7)$ \\
Estilo de vida não saudável & $63,1(58,8-67,3)$ & $84,6(81,3-87,4)$ & $90,2(87,5-92,6)$ & $83,2(81,3-85,0)$ \\
\hline
\end{tabular}

* Valor de $p=0,000$ (teste de qui-quadrado). 
A Tabela 4 apresenta a prevalência de estilo de vida saudável em idosos, adultos e adolescentes do Município de São Paulo, segundo variáveis demográficas e socioeconômicas. Nos idosos, houve uma diferença estatisticamente significante segundo o sexo $(\mathrm{p}=0,0001)$, sendo maior entre as mulheres; segundo a escolaridade em anos de estudos $(\mathrm{p}=0,0245)$, sendo maior entre aqueles com menos anos de estudos; e segundo a raça/cor $(\mathrm{p}=0,0235)$, sendo maior entre os de cor preta. Nos adultos, houve uma diferença estatisticamente significante segundo o sexo ( $\mathrm{p}=0,0015)$, sendo maior entre as mulheres; segundo a escolaridade em anos de estudos $(\mathrm{p}=0,0019)$ e a escolaridade do chefe da família $(\mathrm{p}=$ 0,0092), sendo maior entre aqueles com mais e menos anos de estudos (12 anos ou mais e 0-3 anos). Dentre os adolescentes, não houve diferença estatisticamente significante na prevalência de estilo de vida saudável segundo as variáveis demográficas e socioeconômicas.

A Tabela 5 mostra a prevalência de estilo de vida não saudável em idosos, adultos e adolescentes do Município de São Paulo, segundo os domínios não cumpridos, sendo que os dados apresentados representam as três combinações mais frequentes na amostra. $\mathrm{O}$ consumo alimentar inadequado, avaliado pelo IQD-R, foi o principal domínio responsável pelo estilo de vida não saudável, sendo que $51,5 \%$ dos idosos, $32,2 \%$ dos adultos e 57,9\% dos adolescentes com estilo de vida não saudável não atingiram as recomendações de um consumo alimentar adequado.

Entre os idosos, o não cumprimento das recomendações para um consumo alimentar adequado foi seguido pela combinação do consumo alimentar inadequado e do consumo abusivo de álcool (7,1\%), e pela combinação do consumo alimentar inadequado e do tabagismo (7\%). Entre os adultos, o não cumprimento das recomendações para um consumo alimentar adequado também foi seguido pela combinação do consumo alimentar inadequado e do consumo abusivo de álcool (11,3\%), e pela combinação do consumo alimentar inadequado, do tabagismo e da inatividade física (8,2\%). Dentre os adolescentes, o não cumprimento das recomendações para um consumo alimentar adequado foi seguido pela combinação do consumo alimentar inadequado e da inatividade física (16,7\%), e pela combinação do consumo alimentar inadequado e do consumo abusivo de álcool (10,6\%).

\section{Discussão}

No que diz respeito à atividade física total no Município de São Paulo, o presente trabalho mostrou que $89,2 \%$ dos idosos, 77,2\% dos adultos e 77,2\% dos adolescentes eram ativos. Por outro lado, a pesquisa de Vigilância de Fatores de Risco e Proteção para Doenças Crônicas por Inquérito Telefônico (VIGITEL) de 2012 20, que tem o objetivo de monitorar a frequência e a distribuição dos principais determinantes das DCNT no Brasil por inquérito telefônico, mostrou que a proporção de indivíduos que atingiram a recomendação adequada de atividade física no lazer foi de 47,6\% entre 18 e 24 anos, $39,1 \%$ entre 25 e 34, 31\% entre 35 e 44, 25,8\% entre 45 e 54 anos, 25,2\% entre 55 e 64 e 23,6\% entre 65 anos e mais 21 .

Já ao avaliar a atividade física total, a Pesquisa Nacional de Saúde do Escolar (PeNSE) de 2012 22, que avaliou adolescentes do $9^{\circ}$ ano do Ensino Fundamental, mostrou que a proporção de indivíduos que atingiram a recomendação de atividade física foi de 55\% no Brasil, 55,1\% no Sudeste e 58,7\% no Estado de São Paulo. Assim sendo, esses resultados mostraram que, em todas as faixas etárias, a proporção de indivíduos que atingiram a recomendação de atividade física foi maior na população do presente estudo. Essas diferenças podem ocorrer porque este trabalho avaliou a atividade física total, ou seja, a soma da atividade física no lazer, transporte, trabalho e atividades domésticas. Enquanto isso, o VIGITEL avaliou apenas a atividade física no lazer e o PeNSE avaliou a atividade física total, porém, com base em três domínios que diferem do presente estudo (deslocamento para a escola, aulas de educação física e outras atividades físicas extraescolares). Essas diferenças nos métodos de avaliação podem ocorrer, pois o IPAQ longo permite tanto a avaliação individual de algum domínio específico como a avaliação da atividade física global incluindo todos os domínios, o que pode ocasionar diferentes interpretações dos resultados 11,15,16.

Em relação ao consumo alimentar, este trabalho mostrou que 52,2\% dos idosos, 33,4\% dos adultos e 14,6\% dos adolescentes estavam classificados no terceiro tercil do consumo alimentar, representando uma dieta adequada. Apesar de só avaliar um componente do consumo alimentar para classificar a dieta, o VIGITEL 2012 mostrou que a proporção de indivíduos com consumo recomendado de frutas 


\section{Tabela 4}

Prevalência de estilo de vida saudável em idosos, adultos e adolescentes, segundo variáveis demográficas e socioeconômicas. Inquérito de Saúde no Município de São Paulo (ISA-Capital 2008), São Paulo, Brasil.

\begin{tabular}{|c|c|c|c|}
\hline Variáveis e categorias & $\begin{array}{c}\text { Idosos } \\
\% \text { (IC95\%) }\end{array}$ & $\begin{array}{l}\text { Adultos } \\
\% \text { (IC95\%) }\end{array}$ & $\begin{array}{c}\text { Adolescentes } \\
\%(I C 95 \%)\end{array}$ \\
\hline \multicolumn{4}{|l|}{ Sexo } \\
\hline Feminino & $43,8(39,5-48,3)$ & $19,5(16,3-22,9)$ & $9,3(7,1-12,1)$ \\
\hline Masculino & $26,7(23,0-30,8)$ & $10,3(8,0-13,1)$ & $10,4(8,0-13,3)$ \\
\hline Valor de $\mathrm{p}$ * & 0,0001 & 0,0015 & 0,7190 \\
\hline \multicolumn{4}{|l|}{ Escolaridade (anos de estudo) } \\
\hline $0-3$ & $44,9(40,5-49,3)$ & $23,2(19,8-26,8)$ & - \\
\hline $4-7$ & $37,2(33,1-41,6)$ & $11,1(8,7-14,0)$ & - \\
\hline $8-11$ & $32,7(28,6-36,9)$ & $11,4(8,9-14,2)$ & - \\
\hline 12 ou mais & $20,1(16,8-23,9)$ & $25,2(21,8-29,0)$ & - \\
\hline Valor de $p$ * & 0,0245 & 0,0019 & \\
\hline \multicolumn{4}{|l|}{ Situação conjugal } \\
\hline Casado(a)/União estável & $34,8(30,6-39,0)$ & $15,9(13,1-19,2)$ & $9,8(7,4-12,5)$ \\
\hline Solteiro(a) & $33,1(29,0-37,3)$ & $16,6(13,7-20,0)$ & $6,9(4,9-9,3)$ \\
\hline Separado(a) & $41,8(37,4-46,1)$ & $8,1(6,0-10,6)$ & $9,8(7,4-12,5)$ \\
\hline Viúvo(a) & $41,2(36,9-45,6)$ & $20,0(16,8-23,4)$ & $15,0(12,1-18,2)$ \\
\hline NS/NR & $39,9(35,6-44,2)$ & $17,1(14,2-20,5)$ & 0,0 \\
\hline Valor de $p$ * & 0,6301 & 0,5536 & 0,7314 \\
\hline \multicolumn{4}{|l|}{ Raça/Cor } \\
\hline Branca & $33,0(29,0-37,3)$ & $15,8(12,9-19,0)$ & $11,9(9,3-14,9)$ \\
\hline Preta & $54,8(50,3-59,1)$ & $11,3(8,9-14,2)$ & $3,7(2,4-5,7)$ \\
\hline Parda & $45,2(40,9-49,7)$ & $14,9(12,1-18,1)$ & $8,4(6,3-11,1)$ \\
\hline Outras & $31,3(27,3-35,5)$ & $36,6(32,7-40,7)$ & 0,0 \\
\hline Valor de $\mathrm{p}$ * & 0,0235 & 0,7174 & 0,2573 \\
\hline \multicolumn{4}{|l|}{ Religião } \\
\hline Sem religião & $21,9(18,4-25,8)$ & 0,0 & $10,3(7,9-13,1)$ \\
\hline Católica & $35,9(31,8-40,2)$ & $14,6(11,8-17,7)$ & $9,4(7,1-12,1)$ \\
\hline Protestante/Evangélica & $48,3(44,0-52,8)$ & $19,2(16,1-22,7)$ & $12,4(9,8-15,5)$ \\
\hline Espírita & $23,4(19,9-27,4)$ & $26,2(22,6-29,9)$ & $24,9(21,3-28,7)$ \\
\hline Crê em Deus & $35,2(31,0-39,4)$ & 0,0 & $1,9(1,0-3,5)$ \\
\hline Outras & $39,3(35,0-43,6)$ & $18,3(15,3-21,8)$ & $5,4(3,7-7,6)$ \\
\hline Valor de $p$ * & 0,1939 & 0,0804 & 0,2109 \\
\hline \multicolumn{4}{|c|}{ Renda familiar mensal (salários mínimos) } \\
\hline$<1$ & $41,8(37,4-46,1)$ & $11,7(9,2-14,6)$ & $9,4(7,1-12,1)$ \\
\hline $1-4$ & $35,4(31,2-39,6)$ & $13,7(11,1-16,8)$ & $10,2(7,9-13,1)$ \\
\hline 5 ou mais & $35,3(31,2-39,6)$ & $22,6(19,3-26,3)$ & $9,1(6,9-11,9)$ \\
\hline Valor de $p$ * & 0,5032 & 0,1066 & 0,9413 \\
\hline \multicolumn{4}{|c|}{ Escolaridade do chefe da família (anos de estudo) } \\
\hline $0-3$ & $45,1(40,7-49,5)$ & $21,6(18,4-25,2)$ & $16,5(13,6-19,9)$ \\
\hline $4-7$ & $38,3(34,0-42,6)$ & $9,2(7,0-11,9)$ & $13,3(10,6-16,4)$ \\
\hline $8-11$ & $32,8(28,8-37,1)$ & $12,4(9,8-15,3)$ & $7,8(5,7-10,3)$ \\
\hline 12 ou mais & $28,3(24,5-32,4)$ & $25,1(21,6-28,8)$ & $8,2(6,1-10,9)$ \\
\hline NS/NR & $39,9(35,6-44,2)$ & $17,1(14,2-20,5)$ & 0,0 \\
\hline Valor de $p$ * & 0,2017 & 0,0092 & 0,2141 \\
\hline
\end{tabular}

IC95\%: intervalo de 95\% de confiança; NS/NR: não sabe/não respondeu.

* Valor de p para o teste qui-quadrado. 


\section{Tabela 5}

Prevalência de estilo de vida não saudável em idosos, adultos e adolescente, segundo os domínios não cumpridos que foram mais frequentes na amostra. Inquérito de Saúde no Município de São Paulo (ISA-Capital 2008), São Paulo, Brasil.

\begin{tabular}{|c|c|c|c|c|c|c|}
\hline & \multicolumn{5}{|c|}{ Domínios } & \multirow[t]{2}{*}{ Prevalência (\%) } \\
\hline & Dependência de álcool & Tabagismo & Atividade física & Consumo alimentar & Consumo abusivo de álcool & \\
\hline \multirow[t]{3}{*}{ Idosos } & $\checkmark$ & $\checkmark$ & $\checkmark$ & $x$ & $\checkmark$ & 51,5 \\
\hline & $\checkmark$ & $\checkmark$ & $\checkmark$ & $x$ & $x$ & 7,1 \\
\hline & $\checkmark$ & $x$ & $\checkmark$ & $x$ & $\checkmark$ & 7,0 \\
\hline \multirow[t]{3}{*}{ Adultos } & $\checkmark$ & $\checkmark$ & $\checkmark$ & $x$ & $\checkmark$ & 32,2 \\
\hline & $\checkmark$ & $\checkmark$ & $\checkmark$ & $x$ & $x$ & 11,3 \\
\hline & $\checkmark$ & $x$ & $\checkmark$ & $x$ & $x$ & 8,2 \\
\hline \multirow[t]{3}{*}{ Adolescentes } & $\checkmark$ & $\checkmark$ & $\checkmark$ & $x$ & $\checkmark$ & 57,9 \\
\hline & $\checkmark$ & $\checkmark$ & $x$ & $x$ & $\checkmark$ & 16,7 \\
\hline & $\checkmark$ & $\checkmark$ & $\checkmark$ & $x$ & $x$ & 10,6 \\
\hline
\end{tabular}

X: domínio não cumprido; $\checkmark$ : domínio cumprido.

e hortaliças foi de $17,7 \%$ a $28,5 \%$ entre os adultos, sendo que quanto maior a idade, maior o consumo; e de $28,4 \%$ entre os idosos 20 .

Já um estudo que avaliou os hábitos alimentares de escolares de Pelotas, Rio Grande do Sul, Brasil, entre 13 e 14 anos, mostrou que a proporção de adolescentes com consumo recomendado de frutas e hortaliças foi de 5,3\% 13. Dessa forma, apesar de utilizarem metodologias diferentes, esses resultados mostraram que, em todas as faixas etárias, a proporção de indivíduos que atingiram a recomendação para uma dieta adequada foi maior na população do presente estudo. Porém, os diferentes métodos de avaliação podem interferir nesses resultados, principalmente ao avaliarem a ingestão de frutas e hortaliças, componentes que apresentam baixo consumo em diversos estudos 12,23. Além disso, assim como neste estudo, outras pesquisas mostraram que a qualidade da dieta varia de acordo com a faixa etária, sendo melhor entre os indivíduos mais velhos 23,24. Essa influência da idade sobre a qualidade da dieta pode refletir uma mudança de consciência sobre a saúde com o aumento da idade, interferindo diretamente nas escolhas alimentares pelo grupo mais idoso 23 .

Quanto ao tabagismo no Município de São Paulo, o presente trabalho mostrou que 10\% dos idosos, 25,5\% dos adultos e 4,5\% dos adolescentes eram tabagistas; e 33,3\% dos idosos, 16,5\% dos adultos e 2,6\% dos adolescentes eram ex-tabagistas. Por outro lado, a pesquisa do VIGITEL 2012 mostrou que a proporção de indivíduos tabagistas foi de 8,5\% entre 18 e 24 anos, 11,7\% entre 25 e 34, 12,9\% entre 35 e 44, $16 \%$ entre 45 e 54, 15\% entre 55 e 64 e 7,6\% entre 65 anos e mais. Já a proporção de ex-tabagistas foi de $8,7 \%$ entre 18 e 24 anos, $13 \%$ entre 25 e 34, 16,5\% entre 35 e 44, 30,4\% entre 45 e 54, 39, $1 \%$ entre 55 e 64 e $33,6 \%$ entre 65 anos e mais 20.

O PeNSE 2012 mostrou que a proporção de adolescentes tabagistas do $9^{\circ}$ ano do Ensino Fundamental foi de 5,1\% no Brasil, 5,5\% no Sudeste e 7,3\% no Estado de São Paulo 22. Assim sendo, esses resultados mostraram que a proporção de indivíduos tabagistas foi maior entre os idosos e adultos do presente estudo, mas menor entre os adolescentes. A prevalência de ex-tabagistas foi semelhante entre os idosos e os adultos. Além disso, as menores prevalências de tabagismo entre os mais jovens podem estar relacionadas com o efeito de coorte de nascimento, responsável pela prevalência mais elevada entre os mais velhos, e com o fato de que, em anos recentes, em função de campanhas educativas, medidas regulatórias e restritivas, o tabaco vem perdendo prestígio e adeptos, principalmente nas capitais do Sul e Sudeste.

No que diz respeito ao consumo de álcool no Município de São Paulo, este trabalho mostrou que 3,6\% dos idosos, 4,5\% dos adultos e 2,3\% dos adolescentes eram dependentes de álcool; e 15\% dos idosos, $38 \%$ dos adultos e 18,8\% dos adolescentes referiram um consumo abusivo de álcool. Por outro lado, a pesquisa do VIGITEL 2012 mostrou que a proporção de indivíduos com consumo abusivo de 
álcool foi de $21,8 \%$ entre 18 e 24 anos, 24,7\% entre 25 e 34, $20 \%$ entre 35 e 44, 16,6\% entre 45 e 54 , $11,9 \%$ entre 55 e 64 e $5 \%$ entre 65 anos e mais 20.

Já um estudo que avaliou o consumo abusivo de álcool em adolescentes brasileiros em 2006, mostrou que a proporção deste consumo foi de $24,2 \% 25$. Dessa forma, esses resultados mostraram que a proporção de indivíduos com consumo abusivo de álcool foi maior entre os idosos e adultos da população deste trabalho e menor entre os adolescentes.

Apesar da falta de estudos que utilizam a mesma metodologia para classificar o estilo de vida limitar a comparação com outros trabalhos, é possível dizer que a prevalência de estilo de vida saudável no Município de São Paulo (considerando todo o conjunto de domínios) é baixa, uma vez que só contempla $36,9 \%$ dos idosos, $15,4 \%$ dos adultos e $9,8 \%$ dos adolescentes.

O fato da maior prevalência de estilo de vida saudável estar entre os idosos pode ocorrer por diversos motivos, entre eles está uma maior preocupação com a saúde em consequência do surgimento de doenças ou agravos, o que leva à adoção de hábitos mais saudáveis. Entretanto, isso não explica uma necessidade menor de investimentos em mudanças de hábitos entre idosos, pois os riscos atribuíveis nesta faixa etária são mais altos devido à maior incidência de doenças ou agravos, assim como a maiores riscos devido à perduração dos hábitos. Por outro lado, o fato da menor prevalência de estilo de vida saudável estar entre os adolescentes pode ser explicado pelos atuais hábitos sedentários e de consumo alimentar inadequado, que incluem um tempo sentado elevado e um alto consumo de alimentos processados, presente em grande parte da população mais jovem. Essa situação também exige muito cuidado, pois muitos dos hábitos adquiridos nessa fase da vida são levados para a vida adulta junto com as duas consequências para a saúde 26 .

O estilo de vida apresentou associações significativas com algumas das variáveis demográficas e socioeconômicas estudadas. Dentre os idosos e os adultos, a prevalência de estilo de vida saudável foi maior entre as mulheres. Esses resultados corroboram com estudos que mostram que os homens são mais vulneráveis às doenças e morrem mais precocemente que as mulheres, principalmente por não buscarem cuidados de atenção primária à saúde, o que inclui atividades relacionadas à promoção de um estilo de vida mais adequado 27.

A escolaridade em anos de estudos apresentou uma associação significativa com o estilo de vida, sendo que, entre os idosos, quanto menor a escolaridade, maior a prevalência de estilo de vida saudável. Por outro lado, entre os adultos, as maiores prevalências de estilo de vida saudável estavam tanto entre aqueles com mais anos de estudos como dentre aqueles com menos anos de estudos. Ainda entre os adultos, a prevalência de estilo de vida saudável também foi maior entre aqueles com mais e menos anos de estudos do chefe da família. Esses dados diferem de alguns trabalhos que reforçam a hipótese da determinação social relacionada aos comportamentos de saúde, na qual a escolaridade elevada estaria associada a um estilo de vida mais saudável 21,28. Porém, as escolhas de estilo de vida são comportamentos individuais que envolvem escolhas pessoais. Por essa razão, o estilo de vida relacionado à saúde também pode ser visto como consequência de uma escolha ou responsabilidade individual, e não como reflexo de uma contextualização social ${ }^{29}$, o que poderia explicar as diferenças encontradas nos resultados do presente trabalho.

Entre os idosos houve associações significativas com a raça/cor, sendo que a prevalência de estilo de vida saudável foi maior entre os negros. Apesar de alguns estudos demostrarem hábitos mais saudáveis em brancos 30,31, esse resultado poderia ser explicado pelo fato de, por terem uma maior chance de apresentarem doenças cardiovasculares os negros adotam hábitos mais saudáveis como consequência do surgimento de doenças ou agravos.

Dentre os adolescentes não houve associações significativas entre o estilo de vida saudável e as variáveis demográficas e socioeconômicas estudadas, mostrando que a baixa prevalência de estilo de vida saudável ocorre independentemente do sexo e dos segmentos sociais. Esses resultados também foram observados em outros estudos que mostraram a equalização das desigualdades em saúde na adolescência 32,33.

Atualmente, as teorias de estilo de vida saudável sugerem que as orientações para a saúde são moldadas por importantes fatores sociais, culturais, econômicos, históricos e políticos. Nesse sentido, algumas políticas públicas relacionadas à saúde e ao estilo de vida já existem e estão implantadas no Brasil atualmente, como o Pacto pela Saúde, o Pacto pela Vida, a Política Nacional de Promoção da Saúde, o Programa Academia da Saúde, a Política Nacional de Alimentação e Nutrição, o Programa 
Nacional de Controle ao Tabagismo e a Política de Atenção Integral em Álcool e Outras Drogas. Apesar do presente estudo não ter esse objetivo, os resultados negativos apresentados podem sugerir que essas políticas não estão sendo efetivas para melhorar o estilo de vida da população.

Embora seja um estudo de base populacional, algumas limitações devem ser consideradas, como a falta de padronização das metodologias utilizadas para coletas de dados em inquéritos populacionais e a falta de trabalhos que usaram uma metodologia para classificar o estilo de vida, dificultando a comparação com os resultados de outros estudos. Além disso, todos os fatores de risco foram analisados sem considerar uma ordem de importância na relação com as doenças cardiovasculares.

Com base nos resultados apresentados, foi possível concluir que a prevalência de estilo de vida saudável na população do Município de São Paulo foi baixa, sendo maior entre os idosos, seguida pelos adultos e adolescentes. Dentre os idosos e os adultos, o estilo de vida saudável foi significativamente maior no sexo feminino do que no masculino. Em todas as faixas etárias, o consumo alimentar foi o principal domínio responsável pelo estilo de vida não saudável, seguido pela combinação de consumo alimentar e consumo de álcool entre os idosos e os adultos, e pela combinação de consumo alimentar e atividade física entre os adolescentes. Sendo assim, os resultados deste trabalho evidenciam que a maior parte da população tem um estilo de vida não saudável, mostrando a importância da manutenção das políticas públicas relacionadas à saúde e ao estilo de vida já existentes, ressaltando a necessidade do planejamento de novas estratégias e ações para o controle do estilo de vida não saudável, principalmente relacionadas ao consumo alimentar, e colocando a promoção da alimentação saudável como uma estratégia central para a diminuição da morbimortalidade precoce por doenças cardiovasculares, sendo importante buscar estratégias diferenciadas de acordo com o sexo e a faixa etária.

\section{Colaboradores}

T. K. Ferrari, C. L. G. Cesar, M. C. G. P. Alves, M. B. A. Barros, M. Goldbaum, R. M. Fisberg participaram na concepção e projeto, análise e interpretação dos dados, redação do artigo, revisão crítica relevante do conteúdo intelectual e aprovação final da versão a ser publicada.

\section{Agradecimentos}

À Fundação de Amparo à Pesquisa do Estado de São Paulo (FAPESP; 2009/15831-0) e ao Conselho Nacional de Desenvolvimento Científico e Tecnológico (CNPq; 472873/2012-1) pelo financiamento.

\section{Referências}

1. Monteiro CA, Mondini L, Souza ALM, Popkin BM. Da desnutrição para a obesidade: a transição nutricional no Brasil. In: Monteiro CA, organizador. Velhos e novos males da saúde no Brasil. 2a Ed. São Paulo: Editora Hucitec; 2000. p. 247-55.

2. Alwan A, MacLean DR, Riley LM, d'Espaignet ET, Mathers CD, Stevens GA, et al. Monitoring and surveillance of chronic non-communicable diseases: progress and capacity in high-burden countries. Lancet 2010; 376:1861-8.

3. Botrel TEA, Costa RD, Costa MD, Costa AM. Doenças cardiovasculares: causas e prevenção. Rev Bras Clín Ter 2000; 26:87-90.

4. World Health Organization. Global status report on noncommunicable diseases 2010. Geneva: World Health Organization; 2011.

5. World Health Organization. Noncommunicable diseases country profiles 2011. Geneva: World Health Organization; 2011.

6. Schmidt MI, Duncan BB, Silva GA, Menezes AM, Monteiro CA, Barreto SM, et al. Doenças crônicas não transmissíveis no Brasil: carga e desafios atuais. Lancet 2011; 377:1949-61. 
7. World Health Organization. Global health risks: mortality and burden of disease attributable to selected major risks. Geneva: World Health Organization; 2009.

8. Assah FK, Ekelund U, Brage S, Mbanya JC, Wareham NJ. Urbanization, physical activity, and metabolic health in sub-Saharan Africa. Diabetes Care 2011; 34:491-6.

9. Bazzano LA, Serdula MK, Liu S. Dietary intake of fruits and vegetables and risk of cardiovascular disease. Curr Atheroscler Rep 2003; 5:492-9.

10. Doll R, Peto R, Boreham J, Sutherland I. Mortality in relation to alcohol consumption: a prospective study among male British doctors. Int J Epidemiol 2005; 34:199-204.

11. Florindo AA, Guimarães VV, Cesar CL, Barros MB, Alves MC, Goldbaum M. Epidemiology of leisure, transportation, occupational, and household physical activity: prevalence and associated factors. J Phys Act Health 2009; 6:625-32.

12. Levy-Costa RB, Castro IRR, Cardoso LO, Tavares LF, Sardinha LMV, Gomes FS, et al. Consumo e comportamento alimentar entre adolescentes brasileiros: Pesquisa Nacional de Saúde do Escolar (PeNSE), 2009. Ciênc Saúde Coletiva 2010; 15 Suppl 2:3085-97.

13. Neutzling MB, Assunção MCF, Malcon MC, Hallal PC, Menezes AMB. Hábitos alimentares de escolares adolescentes de Pelotas, Brasil. Rev Nutr 2010; 23:379-88.

14. Oberg M, Jaakkola MS, Woodward A, Peruga A, Prüss-Ustün A. Worldwide burden of disease from exposure to second-hand smoke: a retrospective analysis of data from 192 countries. Lancet 2011; 377:139-46.

15. Hallal PC, Victora CG, Wells JC, Lima RC. Physical inactivity: prevalence and associated variables in Brazilian adults. Med Sci Sports Exerc 2003; 35:1894-900.

16. US Department of Health and Human Services. Physical activity guidelines for Americans. Washington DC: US Department of Health and Human Services; 2008.

17. Previdelli AN, Andrade SC, Pires MM, Ferreira SR, Fisberg RM, Marchioni DM. A revised version of the Healthy Eating Index for the Brazilian population. Rev Saúde Pública 2011; 45:794-8.

18. Moreira LB, Fuchs FD, Moraes RS, Bredemeier M, Cardozo S, Fuchs SC, et al. Alcoholic beverage consumption and associated factors in Porto Alegre, a southern Brazilian city: a populationbased survey. J Stud Alcohol 1996; 57:253-9.

19. Mayfield D, McLeod G, Hall P. The CAGE questionnaire: validation of a new alcoholism screening instrument. Am J Psychiatry 1974; 131:1121-3.

20. Secretaria de Vigilância em Saúde, Ministério da Saúde. VIGITEL 2012. Vigilância de fatores de risco e proteção para doenças crônicas por inquérito telefônico. Brasília: Ministério da Saúde; 2013.
21. Lee IM, Shiroma EJ, Lobelo F, Puska P, Blair SN, Katzmarzyk PT. Effect of physical inactivity on major non-communicable diseases worldwide: an analysis of burden of disease and life expectancy. Lancet 2012; 380:219-29.

22. Instituto Brasileiro de Geografia e Estatística. Pesquisa Nacional de Saúde do Escolar - PeNSE 2012. Rio de Janeiro: Instituto Brasileiro de Geografia e Estatística; 2013.

23. Morimoto JM, Latorre MRDO, Cesar CLG, Carandina L, Barros MBA, Goldbaum M, et al. Fatores associados à qualidade da dieta de adultos residentes na Região Metropolitana de São Paulo, Brasil, 2002. Cad Saúde Pública 2008; 24:169-78.

24. Guo X, Warden BA, Paeratakul S, Bray GA. Healthy eating index and obesity. Eur J Clin Nutr 2004; 58:1580-6.

25. Pinsky I, Sanches M, Zaleski M, Laranjeira R, Caetano R. Patterns of alcohol use among Brazilian adolescents. Rev Bras Psiquiatr 2009; 32:242-9.

26. Salonna F, van Dijk JP, Geckova AM, Sleskova M, Groothoff JW, Reijneveld AS. Social inequalities in changes in health-related behaviour among Slovak adolescents aged between 15 and 19: a longitudinal study. BMC Public Health 2008; 8:57.

27. Silva VLQ. Sexualidade masculina e saúde do homem na estratégia de saúde da família: trabalhando com a equipe a pesquisa-ação [Dissertação de Mestrado]. Ribeirão Preto: Escola de Enfermagem de Ribeirão Preto, Universidade de São Paulo; 2010.

28. Lima-Costa MF. A escolaridade afeta, igualmente, comportamentos prejudiciais à saúde de idosos e adultos mais jovens? Inquérito de Saúde da Região Metropolitana de Belo Horizonte, Minas Gerais, Brazil. Epidemiol Serv Saúde 2004; 13:201-8.

29. Marmot M, Wilkinson RG. Social determinants of health. Oxford: Oxford University Press; 1999.

30. Lima-Costa MF, Matos DL, Camargos VP, Macinko J. Tendências em dez anos das condições de saúde de idosos brasileiros: evidências da Pesquisa Nacional por Amostra de Domicílios (1998, 2003, 2008). Ciênc Saúde Coletiva 2011; 16:3689-96.

31. Oliveira BLCA, Thomaz EBAF, Silva RA. Associação da cor/raça aos indicadores de saúde para idosos no Brasil: um estudo baseado na Pesquisa Nacional por Amostra de Domicílios (2008). Cad Saúde Pública 2014; 30:1-15.

32. Friestad C, Klepp KI. Socioeconomic status and health behavior patterns through adolescence: results from a prospective cohort study in Norway. Eur J Public Health 2006; 16:41-7.

33. Braz M, Barros Filho AA, Barros MBA. Saúde dos adolescentes: um estudo de base populacional em Campinas, São Paulo, Brasil. Cad Saúde Pública 2013; 29:1877-88. 


\section{Abstract}

The objective was to analyze adolescent, adult, and elderly lifestyles in the city of São Paulo, Brazil, according to demographic and socioeconomic variables. A cross-sectional, population-based study was performed with data from the Health Survey in São Paulo City (ISA-Capital 2008) database. Lifestyle was defined on the basis of physical activity, diet, smoking, and alcohol abuse and addiction, according to the respective guidelines. Prevalence of healthy lifestyle was $36.9 \%$ in the elderly, $15.4 \%$ in adults, and $9.8 \%$ in adolescents, and was higher in females in the elderly and adults. Among individuals with unhealthy lifestyle, 51.5\% of the elderly, 32.2\% of adults, and $57.9 \%$ of adolescents failed to reach the guidelines for adequate diet. Prevalence of healthy lifestyle was highest among the elderly, followed by adults and adolescents. Food consumption was the main factor associated with unhealthy lifestyle, demonstrating the importance of interventions to promote healthy lifestyle, especially adequate diet.

Life Style; Motor Activity; Food Consumption; Smoking; Alcoholism

\section{Resumen}

El objetivo fue analizar el estilo de vida de la población adolescente, adulta y anciana del municipio de Sao Paulo, Brasil, de acuerdo con variables demográficas y socioeconómicas. Se realizó un estudio transversal, de base poblacional, utilizando datos del Encuesta de Saúde en el Municipio de São Paulo (ISA-Capital 2008). El estilo de vida fue definido por la evaluación de la actividad física, consumo alimenticio, tabaquismo, consumo abusivo y alcoholismo, de acuerdo con sus respectivas recomendaciones. La prevalencia del estilo de vida saludable fue de un 36,9\% entre ancianos, un $15,4 \%$ entre adultos y un 9, $8 \%$ entre adolescentes, siendo mayor en el sexo femenino entre ancianos $y$ adultos. Entre aquellos con un estilo de vida no saludable, un 51,5\% de los ancianos, un 32,2\% de los adultos y un 57,9\% de los adolescentes no alcanzaron la recomendación para una dieta adecuada. La prevalencia de estilo de vida saludable fue mayor entre ancianos, seguidos por los adultos $y$ adolescentes. El consumo alimentario fue el principal responsable del estilo de vida no saludable, evidenciando la importancia de intervenciones para la promoción del estilo de vida saludable y, principalmente, de una dieta adecuada.

Estilo de Vida; Actividad Motora; Consumo de Alimentos; Hábito de Fumar; Alcoholismo
Recebido em 14/Nov/2015

Versão final reapresentada em 14/Mar/2016 Aprovado em 06/Abr/2016 